\title{
Efficient use of the Noise Budget at Schiphol Airport through Minimax Optimization of Runway Allocations
}

\author{
B.R Kuiper ${ }^{1}$ and H.G. Visser ${ }^{2}$ \\ Delft University of Technology, Delft, the Netherlands \\ S. Heblij ${ }^{3}$ \\ National Aerospace Laboratory NLR, Amsterdam, the Netherlands
}

\begin{abstract}
This paper presents the development of a runway allocation planning tool that seeks to maximize the permissible number of flight operations into and out of an airport within a given annual noise budget. Although the model that underlies the planning tool is generic in nature, the tool has been customized for application to a specific airport, viz. Amsterdam airport Schiphol in the Netherlands. The noise budget regulations applicable at Schiphol stipulate limits on the annual cumulative noise loads at a large number of enforcement points arranged around the airport. To ensure an equitable distribution of the cumulative noise load at the enforcement points, an efficient allocation and distribution of the annual flight movements over available runways and routes is required that takes weather induced restrictions into account. To this end, a Linear Programming (LP) optimization formulation has been developed that implements a minimax performance criterion that aims to minimize the maximum cumulative noise load value occurring at any of the enforcement points. The numerical results obtained for the operational year 2005 clearly demonstrate the potential of the tool to maximize the yearly number of flight movements within the assigned noise budget.
\end{abstract}

\section{Introduction}

$\mathrm{T}$ o mitigate the impact of aircraft noise, an array of measures has been developed and implemented at airports located close to sensitive communities. A type of measure that is frequently employed to mitigate the effect of airport noise concerns the enforcement of airport access restrictions, such as operating quota, noise budgets, (nighttime) curfews and restrictions on the operation of certain (noisy) types of aircraft ${ }^{1}$.

The application of a noise budget generally places a ceiling on the total amount of noise that may be produced at an airport during a longer period such as a season or a year ${ }^{2}$. Due to their long-term nature, noise budgets are typically based on a cumulative (rather than a single-event) noise metric. In some cases noise budgets restrict operations only during a certain night time period, whilst in other cases the budget may apply during the day as well. In contrast to an operating quota, which imposes a direct limit on the permissible number of movements, the application of a noise budget provides some degree of flexibility in the scheduling of operations owing to the fact that it is based on the aggregation and distribution of all flight movements over an extended period of time. For example, within a given noise budget, the operation of a few noisy aircraft might be exchanged in favor of a larger number of quieter planes. By the same token, the permissible number of movements might be increased through efficient allocation and distribution of the annual flight movements over available runways and routes. Noise budget restrictions, presently in place at various noise-sensitive airports around the globe, can take on several forms depending on the community noise policy objectives sought to be achieved at a particular airport.

\footnotetext{
${ }^{1}$ MSc student

2 Associate Professor, Faculty of Aerospace Engineering, P.O. Box 5058, 2600 GB Delft, The Netherlands, Associate Fellow AIAA.

${ }^{3}$ Ph.D. Candidate, Environment and Policy Support, Member AIAA.
} 
At several airports in the UK, including London Heathrow and Manchester airport, a noise budget has been put in place in the form of a "points budget" known as a Quota Count system ${ }^{3}$. Based on ICAO aircraft noise certification data, all arriving and departing aircraft are given a noise classification in terms of a Quota Count (QC), with a noisy aircraft receiving a higher QC than a quieter one. For each inbound and outbound flight, the corresponding QC is then deducted from the points budget that is typically fixed for any given season. This process allows airlines to operate more quiet aircraft in exchange for fewer noisier ones. In Germany, Hamburg airport is subject to noise limits based on the surface area of land around the airport where the noise exposure exceeds a specified $L_{e q}$ energy-equivalent sound level limit $(62 \mathrm{dBA})^{4}$. The airport conducts continuous noise monitoring and reports annually on its operation within the noise budget. At Friedrichshafen Airport in Germany, a similar noise budget restriction is imposed during the six busiest months of the year ${ }^{3}$. Here, a $L_{e q}$ sound level limit of $62 \mathrm{dBA}$ is imposed and monitored at a specific location in a village adjacent to the airport. One of the strictest noise-controlled airports in the United States is Long Beach airport. At Long Beach airport a noise budget has been put in place that aims to have no communities exposed to a community noise equivalent level (CNEL) of $65 \mathrm{dBA}$ or greater $^{3}$. To help enforce the noise budget regulations, the airport has an extensive airport noise and operations monitoring system in place ${ }^{3}$. In New Zealand, noise budgets are imposed at the international airports of Auckland and Wellington. At both airports, outside a certain surface area of land around the airport defined on a map, the daynight average sound level $L_{d n}$ should not exceed $65 \mathrm{dBA}$. At Auckland international airport, an additional area known as Moderate Aircraft Noise Area is defined. At the boundary of this Moderate Aircraft Noise Area $L_{d n}$ should not exceed $60 \mathrm{dBA}$. Noise monitoring is undertaken in the exposed communities ${ }^{3}$.

One of the most noise-sensitive major airports in Europe is Schiphol airport, located near Amsterdam in the Netherlands. To manage the noise resulting from flight operations and to mitigate its worst effects, Schiphol airport and (national and local) authorities have legislated and implemented an extensive set of measures. The noise regulations currently applicable to Schiphol airport, effective since 2003, also include a noise budget restriction that stipulates limits for the total annual noise volume, as well as for the average annual noise exposure at a number of predefined locations in the vicinity of the airport, known as "enforcement points".

Consistent with airline seasonal planning, a noise planning is made for Schiphol airport for an operational year that runs from November 1 through October $31^{5}$. This implies that on October 31 of a given calendar year, the noise exposure that was accumulated as of November 1 of the preceding calendar year should not exceed the regulatory noise limit specified at any of the enforcement points. The annual usage plan, known as the "operational plan", is made well ahead of the start of an operational year and relies on a prediction of the cumulative noise exposure in the enforcement points based on a comprehensive traffic forecast. In the usage plan it must be clearly demonstrated that the environmental impact of the planned operations does not result in a noise budget overrun by year-end. The environmental capacity resulting from the usage plan is then apportioned to airlines through the allocation of slots (date and time of departure or arrival for a single aircraft movement). It is readily clear that in order to make maximum use of the available environmental capacity, the annual flight movements should be efficiently distributed over the available runways and arrival and departure routes such that the annual budget is met at every enforcement point. It is inevitable though that differences between the planned and actual operations will occur during the operational year. Therefore the noise loads produced at the enforcement points are closely monitored during the operational year. When necessary, operational measures are applied to prevent noise budget exceedance.

At present Schiphol airport has five main runways available, permitting a multitude of runway combinations. Typically, three runways are operated simultaneously in a runway configuration, although configurations that feature one, two or four active runways are also possible. However, due to the often changing weather conditions (notably wind speed and direction), the availability of each runway configuration varies. Clearly, any change in runway combination results in a different traffic distribution around the airport and, as a consequence, in a different noise load distribution.

Based on safety and efficiency considerations, runway combinations have been set up and ranked in an order of hierarchy in a preference list by the air traffic service provider at Schiphol airport (LVNL) ${ }^{6}$. The use of an individual runway is determined by the prevailing meteorological conditions and if the weather conditions allow more than one runway combination to be used, then the highest combination from the preference list is selected. Each month, the annual noise load at the enforcement points is recalculated using actual traffic as available and projected traffic for the remaining months. Based on the results, the preference list is updated such as to achieve the most balanced noise load accumulation at the enforcement points without exceeding the noise budget limits set by year-end.

Amsterdam Airport Schiphol has developed an optimization tool that aims to accommodate as many flights as possible during an operational year, while minimizing the chance of exceeding the limit values of the enforcement points ${ }^{5}$. The optimization tool is used in conjunction with a traffic forecast model, a runway combination selection 
model and a collection of historic meteorological datasets. It is readily clear that meteorological data forms an important basis for the traffic and noise distribution forecasts. As mentioned earlier, the steering mechanism presently used in the optimization process to manage the noise load distribution is to allocate over time the order of preference of the available runway combinations.

In Reference 7, an alternative approach to runway allocation optimization is presented. The multi-objective optimization tool proposed in Ref.7, called SNAP (Strategic Noise Allocation Planning), optimizes the allocation of flights to runways on an annual basis with respect to three criteria, viz., noise impact, third party risk and average delay per movement. The multi-objective optimization is also subject to a number of constraints, related to operational procedures, runway capacity and weather conditions. In order to reach a final solution, a trade-off between the three performance criteria needs to be made. The noise criterion considered in SNAP does not relate to average annual noise exposure at predefined locations (i.e., enforcement points), but rather it is based on the number of people expected to be annoyed by annual aircraft noise exposure. In contrast to the optimization algorithm currently in use at Schiphol ${ }^{5}$, which steers the traffic distribution indirectly through adjustment of the preference list, SNAP does not make use of a preference list, but rather directly allocates optimal runway configurations.

The present study on environmental optimization of runway allocations builds on the approach taken in SNAP, by extending it to take into account the current noise regulations that specify limits at discrete enforcement points. To ensure an equitable distribution of the noise load, a minimax performance criterion is adopted. The minimax criterion aims to minimize the risk of exceeding the limit at any enforcement point by minimizing the maximum noise load value occurring at any of the enforcement points ${ }^{6}$. Unlike in SNAP, third party risk and average delay are not considered as performance criteria in the present study. However, these metrics remain available as performance indicators that can be evaluated a posteriori.

\section{Managing the Noise Load Distribution through Runway Allocation}

\section{A. Runway Combination Selection}

In Figure 1 the runway layout at Schiphol airport is presented. Schiphol features six runways in total, including five main runways and one (viz., RWY 04-22) that is primarily used for smaller aircraft and domestic travel. During the day, outside of peak times, the airport often uses one runway for departures and one for arrivals. However, since Schiphol is a hub airport it also needs to accommodate traffic "waves", i.e., peaks in arriving traffic followed by peaks in departing traffic ${ }^{6}$. During an arrival peak, typically two arrival runways are used. Similarly, during a departure peak two departure runways are operated. Occasionally, two departure and two arrival runways are used simultaneously. During the night there is always one runway for arriving traffic, and one runway for departing traffic.

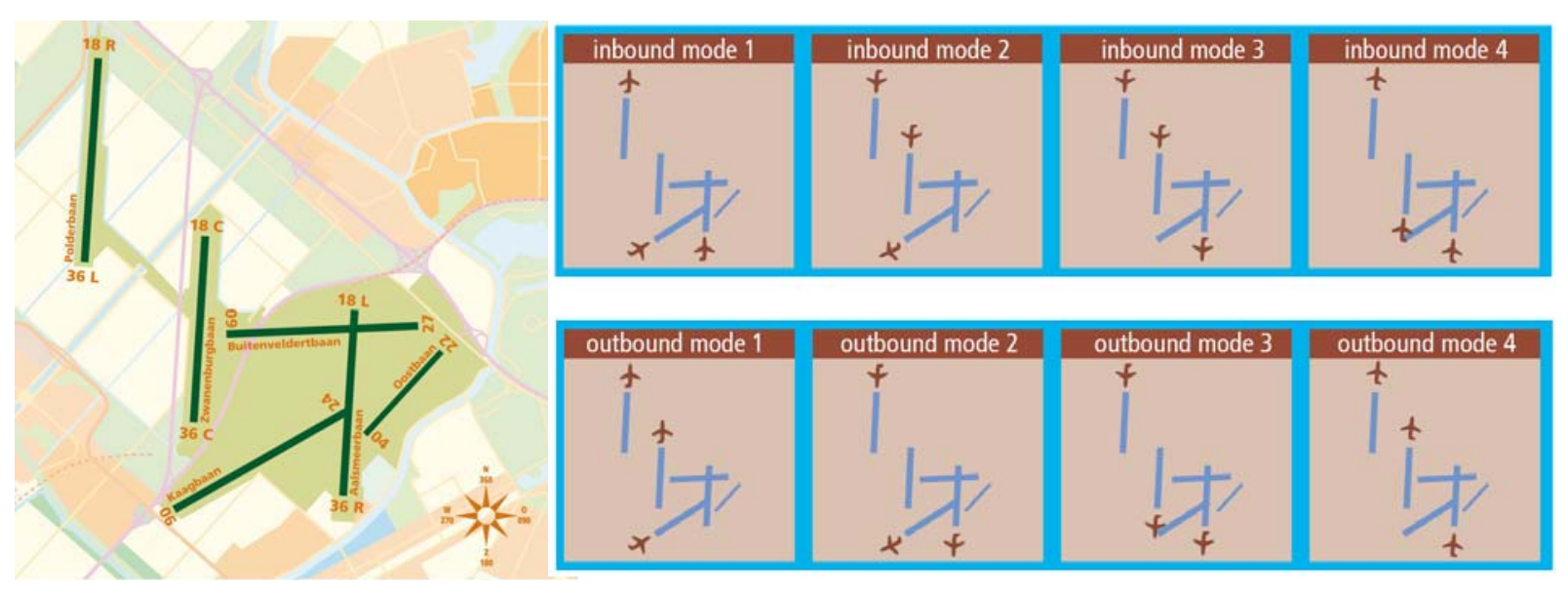

Figure 1: Runway layout (left) and example runway configurations (right) at Schiphol airport. 
Some of the main factors that influence the choice of the best possible runway combination for a particular situation are, peak period and time, visibility, Runway Visual Range (RVR), cloud base and wind limits. In particular the wind limits have a strong influence on which runway combination is most suitable for a particular situation in a particular period of the day. Landings and take-offs are typically conducted into the wind. However, operations on a runway are sometimes permitted with a slight tailwind. The maximum allowable tailwind is usually about 5 to $6 \mathrm{knots}^{8}$. In addition to a tailwind limit, also a limit to the crosswind component is imposed to guarantee flight safety. The crosswind component is the component of the wind velocity vector that is perpendicular the runway centerline. At Schiphol airport, cross wind limits are imposed that depend on the visibility, cloud base and runway surface condition (dry or wet), which affects the braking action ${ }^{9}$.

As mentioned earlier, predictions of future runway allocations and associated noise load distributions are based on an average of past years meteorological data. Historical wind statistics can be conveniently summarized in the graphical form of a so-called wind $\operatorname{rose}^{8}$ (see Figure 2). In essence, a wind rose arranges velocity, direction, and frequency of wind occurrences within a certain period of time. Circles on the template represent the wind speed, while the radial lines illustrate the angles or the wind blowing directions. Each cell bounded by two circle segments and two radial lines contains the percentage of time that the winds correspond to a given direction and velocity range. On the wind rose shown in Figure 2, a runway template (rectangle shown in red) is placed, representing a one-directional runway. The location, dimensions and orientation of the rectangle are determined by the direction of operation and by the applicable cross and tail wind limits. All wind conditions within the rectangle are essentially covered by the considered runway. It is important to realize that for any given runway configuration to be valid under a particular wind condition, that wind condition (cell) should be contained within the "box " associated to each individual runway available in that configuration.

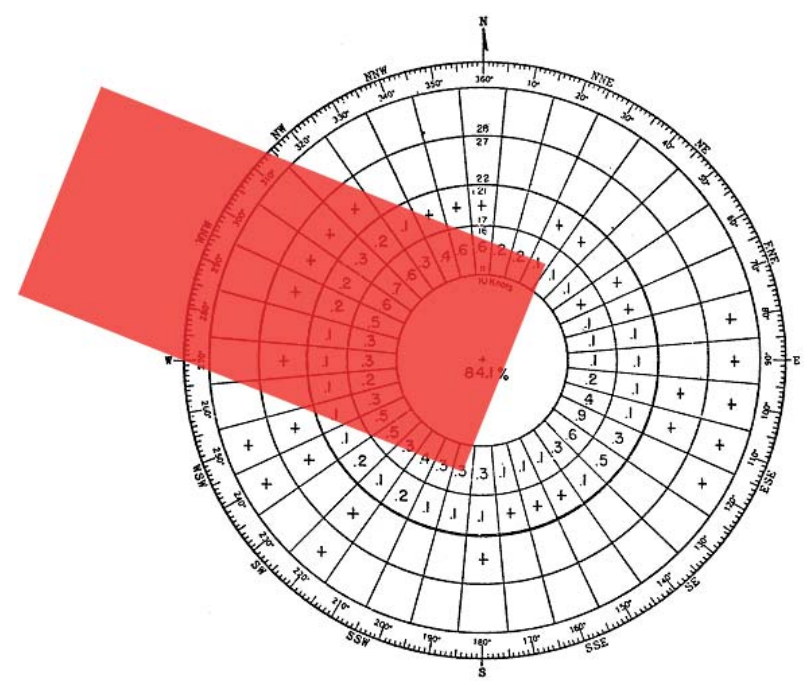

Figure 2: A wind rose and superimposed one-directional runway wind limit template.

\section{B. Runway Allocations}

Runway allocation is the process of assigning a runway to each flight movement. Clearly, runway allocation is to a large extent implicitly determined by the runway combination selection process. Only for configurations that feature multiple runways for departure or landing operations, the runway allocation process also involves distribution of flights over the different runways available in the configuration. When a flight is assigned to a particular runway, the spatial allocation of the noise impact associated to that flight is to a large extent fixed as well. Indeed, the origin or destination of a flight, in combination with the selected runway, usually results in a particular 
routing to or from the airport. It is exactly this property that makes runway allocation such an important steering mechanism with respect to noise management.

\section{Noise Enforcement Points}

To limit the noise disturbance for the population around Schiphol, the Dutch government has specified limits for yearly day-evening-night average sound level $L_{d e n}$ in 35 enforcement point located in the vicinity of the airport (see Figure 3$)^{5}$. A specific $L_{\text {den }}$ limit value has been assigned to each individual enforcement point. The noise limit values for the enforcement points are listed in the table displayed in Figure 3 . It needs to be noted that $L_{d e n}$ is a cumulative measure of noise that expresses the total noise effect at a given location of all the aircraft movements taking place within an operational year. The metric $L_{\text {den }}$ features weightings of the single event levels depending on the time of day or night at which they occur. In addition to the limits on yearly $L_{d e n}$ at the 35 enforcement points, also yearly $L_{\text {night }}$ (applicable to the night period, 23:00 - 07:00 hr. local time) limits have been imposed at 25 separate enforcement points close to the airport.

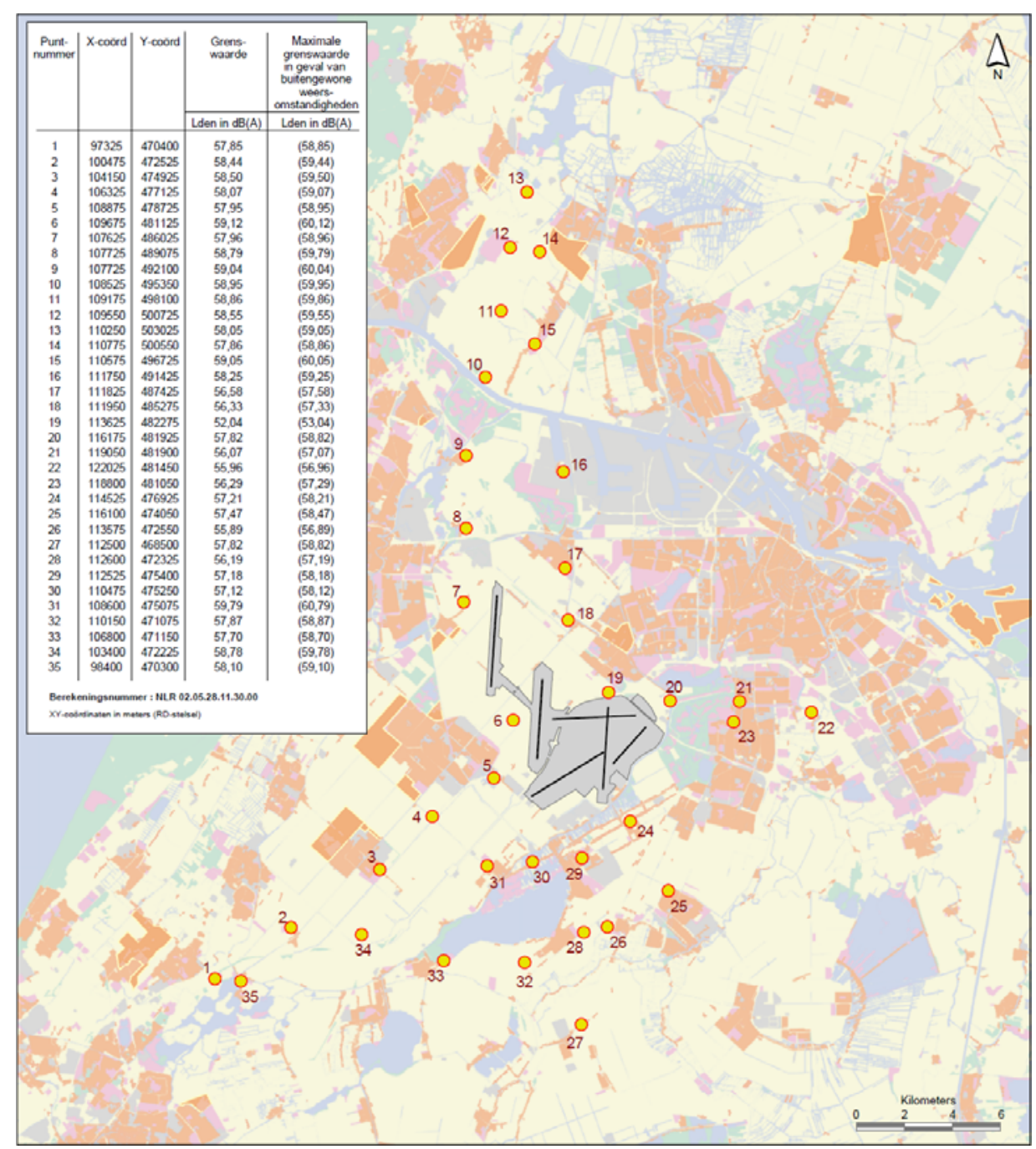

Figure 3: the 35 enforcement points $\left(L_{d e n}\right)$ in the vicinity of Schiphol airport (Ref. 6). 


\section{Minimax Formulation of the Runway Allocation Problem}

\section{Conceptual model description}

In this section a concept description is given of a model that forms the basis for a tool that optimizes the runway allocation for the planned flight movements within an operational year, using a Linear Programming (LP) formulation ${ }^{10}$. The main inputs for the model are identified wind patterns and. traffic patterns.

In a first step, a wind rose analysis is conducted as described in section II.B, using the weather history from the year 1951 until $2005^{7}$. The outcome of this analysis is a list containing for each cell of the wind rose the historic occurrence percentage of the associated wind condition. Next, for each cell in the list it is determined which runway combinations are available, based on the prevailing cross and tail wind limitations. All cells that share the same list of available runway configurations are clustered in a "wind pattern". For Schiphol airport only 19 unique wind patterns were identified ${ }^{6}$.

In a next step, the traffic patterns and their associated noise load distributions are determined. Based on the flight schedule for the operational year (also taking into account projected charter operations) it is determined for each hour of the year how many arrivals and departures take place and by what aircraft type. With 365 x $24=8760$ hours in a year, this gives 8760 traffic samples. To limit the scale of the optimization problem to be formulated, similar traffic samples are aggregated into "traffic patterns". In this context "similar" is defined as, (i) the same number of departures, (ii) the same number of arrivals, and, (iii) same period of the day (Day, Evening, Night). The structure of a traffic pattern could, for example, look like:

\section{Pattern 33 :}

Arrivals: 10

Departures: 7

Traffic sample occurrence: $3 \mathrm{x}$

Arriving a/c: 12x B747, 14x B737, 4x A320

Departing a/c: 10x B737, 9x A320, 2x MD11

Period of the day: evening

When for example the 2005 schedule of Schiphol is considered, this approach aggregates the 8760 yearly traffic samples into about 2000 unique traffic patterns. One of the advantages of the selected form of aggregation is that it preserves the capability to assess average runway delays, which depends on the number of traffic movements per hour and the traffic mix. Also note that specific information regarding the time of day is needed to enable $L_{d e n}$ noise load calculations at the enforcement points.

In the model formulation, each traffic pattern is combined with each wind pattern, resulting in a list of unique combined "situations" (see figure 4). This list represents every situation that can occur during a year, combined with the probability that it occurs. In the optimization formulation, an allocation decision for each of these unique situations has to be made. Here, a decision comes down to the choice of one of the available runway configurations, and in the case of multiple arrival/departure runways also the percentages of distribution over the two runways. For each unique combined situation it now has to be checked which of the available runway configurations have sufficient capacity to handle the traffic. Runway configurations that are available but supply insufficient capacity are discarded. After this, a check is made whether there are some situations with no available runway configurations at all. If so, these unique situations are removed from the model to prevent that the LP problem formulation becomes infeasible.

To choose the optimal runway configuration, the LP problem formulation needs to contain information on the consequence of each allocation choice. Therefore the noise cost for the usage of each available runway configuration is determined for each unique combined situation. Note that the noise contribution values have to be determined separately for each enforcement point, since the noise load accumulation in each enforcement point needs to be recorded. In this study the methodology for conducting the noise load calculations has been principally based on the approach taken in $\mathrm{SNAP}^{7}$.

The SNAP tool relies on the Integrated Noise Model (INM) ${ }^{11}$ to record the noise load contribution at each noise observation point, for each individual flight movement. The results for the single event flight movement noise computations are provided in terms of Sound Exposure Levels (SEL). The spatial distribution of noise caused by a departing or approaching aircraft depends not only on the assigned runway, but also on the route leading to the runway (when considering an arrival) or the route leading from the runway (when considering a departure). In total, 104 routes have been defined for Schiphol airport ${ }^{6}$. To calculate the noise of a flight movement, a flight path is 
synthesized by combining one of the existing departure/arrival tracks, with a standard INM profile (vertical flight path) for the considered aircraft type. It needs to be noted that for the purpose of noise calculations, each aircraft type in the yearly flight schedule is substituted by one of 15 standard types, representing various noise categories. The calculated noise levels, stored in a data file, are thus unique for a certain aircraft type, a certain flight procedure (arrival or departure), and a given runway in conjunction with one of the associated tracks. The noise cost for the usage of each available runway configuration can now be determined upfront for each unique combined situation through aggregation of the individual flight movement results. In the case that a runway configuration features multiple departure and/or arrival runways, the noise cost for the usage of each individual runway needs to be assessed. More specifically, the noise cost for each possible mode of a configuration needs to be determined. In this context, a mode is a combination of a specific departure and a specific arrival runway of a certain configuration. Table 1 gives the possible modes of each of the four configuration types considered ${ }^{6}$.

Table 1: The possible modes for each configuration type $(\mathrm{A}=$ Arrival; $\mathrm{D}=$ Departure $)$.

\begin{tabular}{ll}
\hline Configuration Type & Available Modes $(k)$ \\
\hline 1A + 1D & (1) single departure runway combined with arrival runway \\
2A + 1D & (1) arrival runway 1 combined with the single departure runway \\
& (2) arrival runway 2 combined with the single departure runway \\
1A+ 2D & (1) departure runway 1 combined with the single arrival runway \\
& (2) departure runway 2 combined with the single arrival runway \\
2A+2D & (1) departure runway 1 combined with arrival runway 1 \\
& (2) departure runway 1 combined with arrival runway 2 \\
& (3) departure runway 2 combined with arrival runway 1 \\
& (4) departure runway 2 combined with arrival runway 2 \\
\hline
\end{tabular}

When determining the noise cost of an allocation choice, it is assumed that each departure/arrival route connected to a specific runway is used equally often on average. Therefore, the noise cost is the average of the noise cost calculated for each departure/arrival track separately.

In the present model runway delay is neither an optimization criterion nor an operational constraint. Instead, a constraint on runway throughput capacity has been introduced. Moreover, the possibility exists to perform delay calculations a posteriori once the optimization process has been completed, thus allowing to assess the delay consequences of the allocation choices that have been made. The model will calculate the arrival and departure delay for all the allocated traffic, and subsequently takes the weighted average. The delay calculations are based on a steady state $\mathrm{M} / \mathrm{G}$ queuing model as presented in Ref.7.

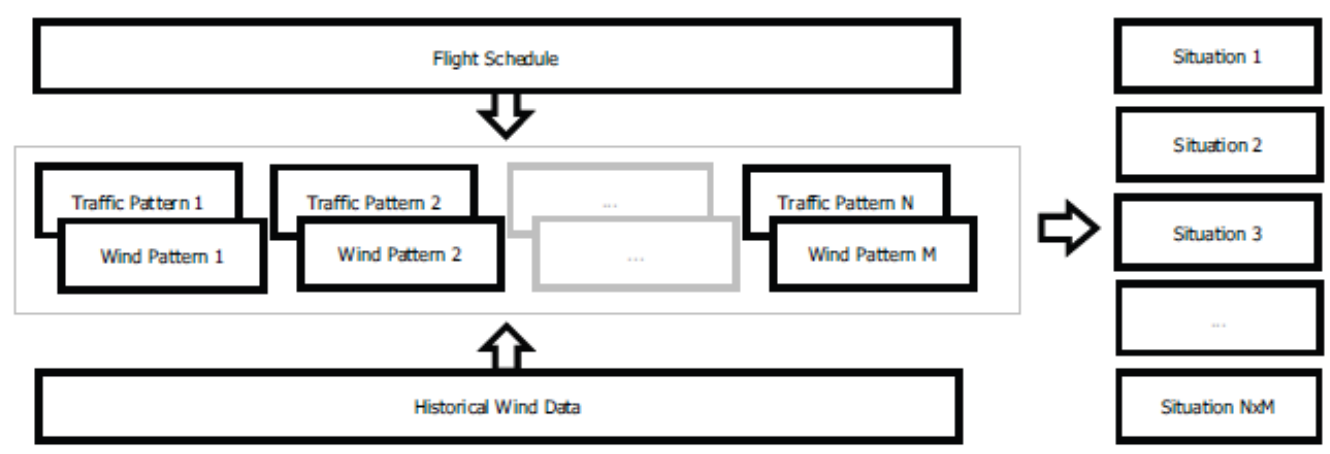

Figure 4: schematic view of the modeling approach. 


\section{E. Performance criteria}

As outlined previously, the noise regulations applicable to Schiphol airport stipulate noise limits for yearly dayevening-night average sound level $L_{d e n}$ at 35 enforcement points. The $L_{d e n}$ value at a given location for a certain period can be calculated using the following equation ${ }^{12}$ :

$$
L_{\text {den }}=10 \cdot \log \left[\sum_{n=1}^{n_{\text {fights }}} w_{n} 10^{\frac{S E L_{n}}{10}}\right]-10 \cdot \log \left[\frac{T_{\text {den }}}{\tau}\right] \text {, }
$$

where $n_{\text {flights }}$ is the number of flight movements within the considered period $T_{\text {den }}, S E L_{n}$ is the Sound Exposure Level of the $n^{\text {th }}$ flight, $w_{n}$ is an adjustment factor tor the $n^{\text {th }}$ event (enabling to penalize movements occurring during the evening or night), and $\tau$ is a reference period of one second.

The fact that the $L_{d e n}$ metric is logarithmic in nature hampers its direct use in a linear optimization formulation. For this reason, rather than to consider the $S E L$ value of a flight movement, its associated acoustic energy level is considered:

$$
\frac{E_{n}}{E_{0}}=10^{\frac{S E L_{n}}{10}}
$$

where $E_{0}$ is a reference sound exposure. Substitution of Eq.(2) into Eq.(1) yields:

$$
L_{\text {den }}=10 \cdot \log \left[\sum_{n=1}^{n_{\text {fights }}} w_{n} \frac{E_{n}}{E_{0}}\right]-10 \cdot \log \left[\frac{T_{\text {den }}}{\tau}\right]
$$

Instead of using $L_{d e n}$, cumulative acoustic energy:

$$
\left[\sum_{n=1}^{n_{\text {fights }}} w_{n} \frac{E_{n}}{E_{0}}\right]
$$

is now addressed in the optimization formulation.

In this study two distinct energy-based performance criteria have been considered. The first criterion aims to minimize the average energy at an enforcement point through the minimization of the summated energies of the noise at all the enforcement points. This principle is illustrated in Figure 5a. The second objective function is a minimax criterion that aims to minimize the maximum noise load value (in terms of cumulative energy) occurring at any of the enforcement points. In practical terms the minimax criterion has been implemented as the minimization of a limit value $L$, where $L$ is always larger or equal than the noise load at any individual enforcement point. The minimax principle is displayed in Figure 5b.

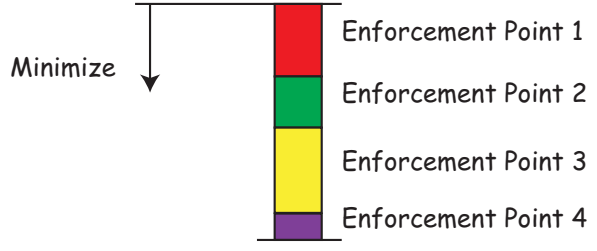

(a) minimum average energy

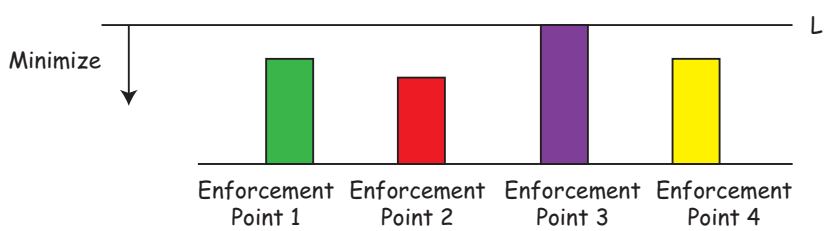

(b) minimum energy of worst individual

Figure 5: illustration of the two energy-based performance criteria. 


\section{F. Mathematical model}

In this section, the LP model for the noise allocation problem is defined.

\section{Decision variables}

The variables that are used to define the noise allocation problem are.

$\begin{array}{lll}\text { real } x_{i, j} & 0 \leq x_{i, j} \leq 1 & \begin{array}{l}\text { fraction of instances that configuration } j \text { is selected in situation } i \\ \text { fraction of the traffic assigned to mode } k \text { of configuration } j \text { for situation } i\end{array} \\ \text { real } w_{i, j, k} & 0 \leq w_{i, j, k} \leq 1 & \begin{array}{l}\text { minimax criterion, expressed as a fraction of the imposed cumulative } \\ \text { real } L\end{array} \\ 0 \leq L \leq 1 & & \end{array}$

By defining $X_{i, j}$ as a real variable, rather than as a binary variable, it may occur that multiple configurations can be assigned in a given situation $i$. Suppose that situation $i$ occurs 10 times in an operational year. A value $x_{i, j}=0.7$ then implies that in seven out of the ten instances that situation $i$ occurs, configuration $j$ is selected.

\section{Cost coefficients}

The coefficients entered in the performance criteria are:
$C_{i, j, k, l}$ noise cost at enforcement point $l$ for mode $k$ of configuration $j$ in situation $i$
$p_{i}$ the occurrence percentage of situation $i$
$q_{i}$ the $D E N$ (Day-Evening-Night) multiplier for situation $i$

\section{Constraints}

The constraints applicable to the noise allocation problem are:

(i) Configuration choice:

$$
\sum_{j=1}^{j=s_{i}} x_{i, j}=1, \quad \forall i=1, . ., r
$$

where $r$ is the total number of situations considered, and $S_{i}$ the maximum number of configurations available for situation $i$. The configuration choice constraint ensures that in each instance in which a particular situation occurs exactly one runway configuration is assigned.

(ii) Runway distribution:

$x_{i, j}=\sum_{k=1}^{k=t_{j}} w_{i, j, k} \quad, \quad \forall i=1, . ., r, \quad \forall j=1, . ., s_{i}$

where $t_{j}$ is the maximum number of modes available for runway configuration $j$. The runway distribution constraint ensures that the traffic fractions summated over all modes $k$ of a configuration $j$ in situation $i$ do not exceed the total traffic assigned to configuration $j$ in situation $i$.

(iii) Runway capacity:

(a) Applicable for runway configurations of the type 2A + 1D and 1A + 2D (see Table 1): 


$$
\begin{array}{ll}
w_{i, j, k} A_{i} \leq 0.9 C_{A} x_{i, j}, & \forall i=1, \ldots, r, \quad k=1,2 \\
w_{i, j, k} D_{i} \leq 0.9 C_{D} x_{i, j} \quad, \quad \forall i=1, . ., r, & k=1,2
\end{array}
$$

(b) Applicable for runway configurations of the type 2A + 2D (see Table 1):

$$
\begin{array}{ll}
\left(w_{i, j, 1}+w_{i, j, 3}\right) A_{i} \leq 0.9 C_{A} x_{i, j}, & \forall i=1, . ., r \\
\left(w_{i, j, 2}+w_{i, j, 4}\right) A_{i} \leq 0.9 C_{A} x_{i, j} & , \quad \forall i=1, . ., r \\
\left(w_{i, j, 1}+w_{i, j, 2}\right) D_{i} \leq 0.9 C_{D} x_{i, j}, & \forall i=1, . ., r \\
\left(w_{i, j, 3}+w_{i, j, 4}\right) D_{i} \leq 0.9 C_{D} x_{i, j}, & \forall i=1, . ., r
\end{array}
$$

where $A_{i}$ and $D_{i}$ are, respectively, the amount of arriving and departing aircraft in situation $i, C_{A}$ and $C_{D}$ are, respectively, the arrival and departure throughput capacity for a single runway. A $90 \%$ load factor has been defined to reduce the theoretical capacity limit into a practical capacity limit. The runway capacity constraints are applied only to runway configurations that feature multiple departure and/or arrival runways. The runway constraints preclude traffic overloading on a given runway. Note that constraints (9) and (11) relate to runway 1, whilst constraints (10) and (12) are associated to runway 2 of the $2 \mathrm{~A}+2 \mathrm{D}$ runway configuration. It is recalled that no constraints are needed for the overall configurations, since configurations with insufficient capacity for a given situation were already removed from the list of available configurations upfront.

(iv) Noise load at enforcement points (only applicable in conjunction with the minimum average energy criterion):

(a) Applicable in conjunction with the minimum average energy criterion:

$$
\sum_{i=1}^{i=r} p_{i} q_{i} \sum_{j=1}^{j=s_{i}} \sum_{k=1}^{k=t_{j}} c_{i, j, k, l} w_{i, j, k} \leq \operatorname{Lim}_{l}, \quad \forall l=1, \ldots, v
$$

where $v$ is the total number of enforcement points, and $\operatorname{Lim}_{l}$ is the imposed noise load limit at enforcement point $l$. The noise load constraints evaluate the cumulative noise loads in the enforcement points and force the resulting values to be smaller than the imposed limits.

(b) Applicable in conjunction with the minimax criterion:

$$
\sum_{i=1}^{i=r} p_{i} q_{i} \sum_{j=1}^{j=s_{i}} \sum_{k=1}^{k=t_{j}} c_{i, j, k, l} w_{i, j, k} \leq L \cdot \operatorname{Lim}_{l}, \quad \forall l=1, \ldots, v
$$

The noise constraint (14) is similar in behavior to constraint (14), except that the permissible value at the enforcement point is reduced by a factor $L$, a value we seek to minimize in the minimax criterion.

\section{Objective functions}

(a) Minimum average cumulative energy criterion: 
$\min Z=\sum_{i=1}^{i=r} p_{i} q_{i} \sum_{j=1}^{j=s_{i}} \sum_{k=1}^{k=t_{j}} \bar{c}_{i, j, k} w_{i, j, k} \quad$,

where:

$$
\bar{c}_{i, j, k}=\sum_{l=1}^{l=v} c_{i, j, k, l} \quad, \quad \forall i=1, . ., r, \quad \forall j=1, . ., s_{i}, \quad \forall k=1, . ., t_{j}
$$

is the noise cost for mode $k$ of configuration $j$ in situation $i$ aggregated over all enforcement points.

(b) Minimax criterion:

$$
\min Z=L
$$

To handle the noise allocation problem outlined above, Linear Programming (LP), one of the most commonly employed operations research methods for large-scale problems, has been successfully used ${ }^{10}$. More specifically, a commercial LP package called CPLEX has been employed ${ }^{13}$.

\section{A Case Study for Schiphol Airport}

\section{A. Assumptions}

To illustrate the developed concept, a case study is presented pertaining to the operational year 2005. Processing of the flight schedule resulted in about 2000 unique traffic patterns. Similarly, processing of the historic wind data produced 19 unique wind patterns. The historic wind data set that has been used to predict the wind conditions for 2005, covers the measurement period from 1951 to 2005 (over 460000 hours). Combination of the identified wind and traffic patterns leads to about 38000 unique situations, to each of which a runway configuration and mode assignment needs to be made. In the model 30 different runway configurations have been considered for Schiphol airport, including $131 \mathrm{~A}+1 \mathrm{D}$ configurations, $82 \mathrm{~A}+1 \mathrm{D}$ configurations, $81 \mathrm{~A}+2 \mathrm{D}$ configurations and $12 \mathrm{~A}+2 \mathrm{D}$ configuration. As an illustration, Table 2 lists the available configurations of the type $2 \mathrm{~A}+1 \mathrm{D}$.

Table 2: Double arrival single departure runway configurations at Schiphol airport.

\begin{tabular}{cccc}
\hline Configuration & Arrival 1 & Arrival 2 & Departure \\
\hline 14 & 06 & $36 \mathrm{R}$ & $36 \mathrm{~L}$ \\
15 & $18 \mathrm{R}$ & $18 \mathrm{C}$ & 24 \\
16 & $18 \mathrm{R}$ & $18 \mathrm{C}$ & $18 \mathrm{~L}$ \\
17 & 27 & $36 \mathrm{C}$ & $36 \mathrm{~L}$ \\
18 & 27 & $18 \mathrm{R}$ & 24 \\
19 & $18 \mathrm{R}$ & 22 & 24 \\
20 & $18 \mathrm{R}$ & 22 & $18 \mathrm{~L}$ \\
21 & 06 & 09 & 09 \\
\hline
\end{tabular}

Combined with the fact that 35 enforcement points need to considered, it is readily clear that a very large scale optimization problem results. To keep the computational burden within acceptable limits, the number of enforcement points taken into account in the optimization was reduced from 35 to 9 in this study. The 9 points were selected such that no runway could be used unrestricted without contributing to the noise load development. This led to the choice of the 9 enforcement points that are displayed in Figure 6. It is readily clear that the use of only 9 enforcement points rather out of the 35 that are actually imposed, implies a limitation of the model. Because the noise load accumulation in the remaining 26 enforcement points is not accounted for, there is a larger degree of freedom in the allocation process, which may potentially lead to different allocation choices. Of course, it is possible 
to a posteriori evaluate the actual noise load accumulated in those 26 remaining enforcement points, allowing to detect possible anomalies.

In addition to the reduced number of enforcement point taken into account, several other limitations of the model need to be mentioned. First of all, the model entirely ignores the 25 enforcement points used during the night time hours. Also, reduced visibility conditions, which influence the availability of runway configurations, have not been taken into account. Another limitation relates to the flight schedule. The original schedule does not include charter operations and to allow for this the charter flights actually recorded for 2005 have been artificially added to the schedule and distributed evenly over the year. In reality this is probably not the case. It needs to be noted that, in principle, most of the above limitations can be removed in follow-on studies.

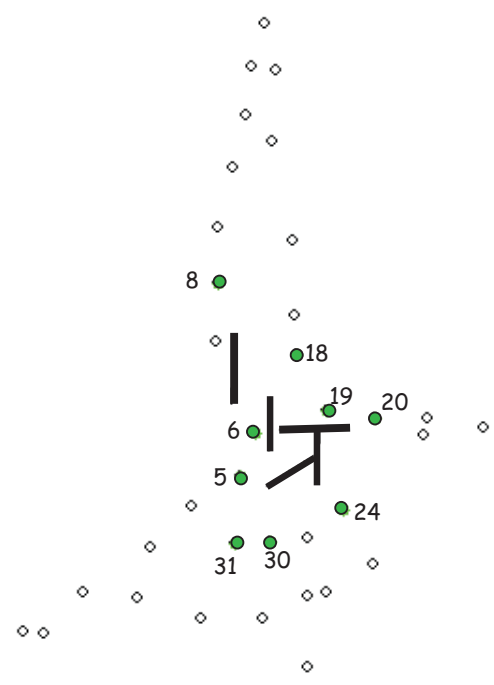

Figure 6: The nine enforcement points incorporated in the optimization model.

\section{B. Optimization results}

In Figure 7 the optimization results are displayed for both the minimum average energy criterion and the minimax criterion for the operational year 2005 .

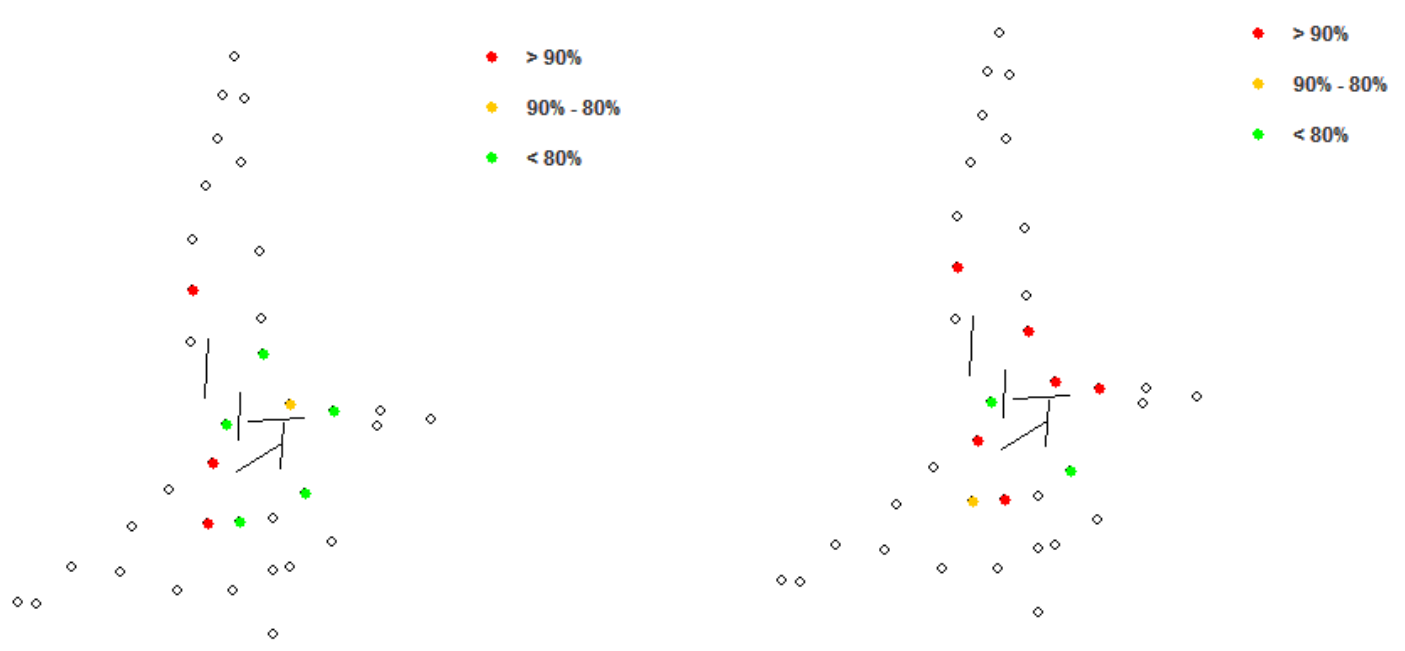

Figure 7: The results for the year planning for 2005 for, minimum average energy criterion (left), and minimax criterion (right) 
Tables 3 and 4 summarize the numerical results for, respectively, the minimum average energy criterion and the minimax criterion. As can be seen from Figure 7 and Table 3, in the minimum average energy solution, the traffic is concentrated in the area south-west from the "Kaagbaan" (runway 06-24), and to the north of the "Polderbaan" (18R-36L). For the Polderbaan this is probably caused by the fact that no enforcement points are located near the approach/departure path of this runway, resulting in only a moderate contribution of flight movements to the objective function. For the Kaagbaan the reason is probably that during certain weather situations (i.e. wind from the west or south-west), no other runways are available. It can be seen that relatively few flights are assigned to the "Buitenveldertbaan." (09-27). This was to be expected due to the fact that enforcement points are placed in the direct vicinity of the runway, thus contributing significantly to the objective function. This behavior closely resembles reality, where the Polderbaan and Kaagbaan are the preferential runways, whilst the Buitenveldertbaan is avoided as much as possible.

Table 3: Optimal planning results for the minimum average energy criterion.

\begin{tabular}{lccc}
\hline Location & $L_{d e n}$ Limit Value $(\mathrm{dB})$ & $L_{d e n}$ ActualValue $(\mathrm{dB})$ & Percentage (\%) \\
\hline Point 5 & 57.71 & 57.71 & 100 \\
Point 6 & 59.03 & 55.91 & 48.8 \\
Point 8 & 57.98 & 57.98 & 100 \\
Point 18 & 58.78 & 54.79 & 39.9 \\
Point 19 & 51.89 & 51.40 & 89.3 \\
Point 20 & 57.57 & 50.15 & 18.1 \\
Point 24 & 56.96 & 54.37 & 55.1 \\
Point 30 & 56.87 & 52.82 & 39.4 \\
Point 31 & 59.54 & 59.31 & 94.8 \\
\hline
\end{tabular}

Table 4: Optimal planning results for the minimax criterion.

\begin{tabular}{lccc}
\hline Location & $L_{d e n}$ Limit Value $(\mathrm{dB})$ & $L_{d e n}$ Actual Value $(\mathrm{dB})$ & Percentage (\%) \\
\hline Point 5 & 57.71 & 57.33 & 91.5 \\
Point 6 & 59.03 & 57.22 & 65.9 \\
Point 8 & 57.98 & 57.60 & 91.5 \\
Point 18 & 58.78 & 58.40 & 91.5 \\
Point 19 & 51.89 & 51.51 & 91.5 \\
Point 20 & 57.57 & 57.19 & 91.5 \\
Point 24 & 56.96 & 54.70 & 59.4 \\
Point 30 & 56.87 & 56.49 & 91.5 \\
Point 31 & 59.54 & 58.98 & $\mathbf{8 7 . 8}$ \\
\hline
\end{tabular}

From Table 4 and Figure 7 it can be seen that for the minimax solution the traffic is more spread out over the various runways to realize balanced noise load development. The enforcement points are kept below their limit values as much as possible. To realize this, runways that are not noise preferential also have to be used. This actually leads to a higher overall cumulative noise value (higher average energy value at the enforcement points). This is demonstrated in Table 5, which compares the characteristic values of the two results. Note that in this comparison the cumulative energy levels obtained for the two criteria have been expressed as a ratio relative to the total noise energy value that was actually recorded at Schiphol airport in $2005^{6}$.

The results in Table 5 clearly reveal that whilst the use of the minimum average energy criterion results in a cumulative energy level that is significantly lower than the actually realized result (about 15\%), the cumulative energy level is considerably higher than the actually realized result when the minimax planning approach is used (some 10\%). This appears to be realistic. Since Schiphol aims to realize a balanced development of noise load by spreading the traffic, it relies on an approach similar in nature to the minimax approach. However, the actual noise spreading will never be as balanced as in the minimax approach taken here, simply because the minimax optimization approach does not rely on (i.e., is not constrained by the use of) a preference list, but rather always directly allocates the optimal configurations. In addition, the extended freedom that the minimax model has by not considering all 35 enforcement points also allows it to accumulate the noise loads in a more balanced fashion. 
Table 5: Comparison of the optimal planning results for the two performance criteria.

\begin{tabular}{ccccc}
\hline Objective & $\begin{array}{c}\text { Total Noise } \\
\text { Energy Ratio }\end{array}$ & $\begin{array}{c}\text { Av. Departure } \\
\text { Delay (min.) }\end{array}$ & $\begin{array}{c}\text { Av. Arrival } \\
\text { Delay (min.) }\end{array}$ & $\begin{array}{c}\text { Solution Time } \\
\text { (min.) }\end{array}$ \\
\hline Av. Energy & $\mathbf{0 . 8 4 6}$ & $\mathbf{0 . 9 6}$ & 2.30 & 17 \\
Minimax & 1.105 & 0.99 & 2.46 & 102 \\
\hline
\end{tabular}

Table 5 also includes results pertaining to average delay. It can be seen from the table, that the average delay values do not differ very much for both cases. There is marked difference between the two cases with respect to the required computational effort. Indeed, the calculation time needed to establish a minimax solution is significantly higher for the minimax formulation.

Finally, in Figure 8 a comparison is given of the noise loads at the enforcement points obtained for the two optimization cases and the actual noise load realized at Schiphol airport in the operational year 2005.

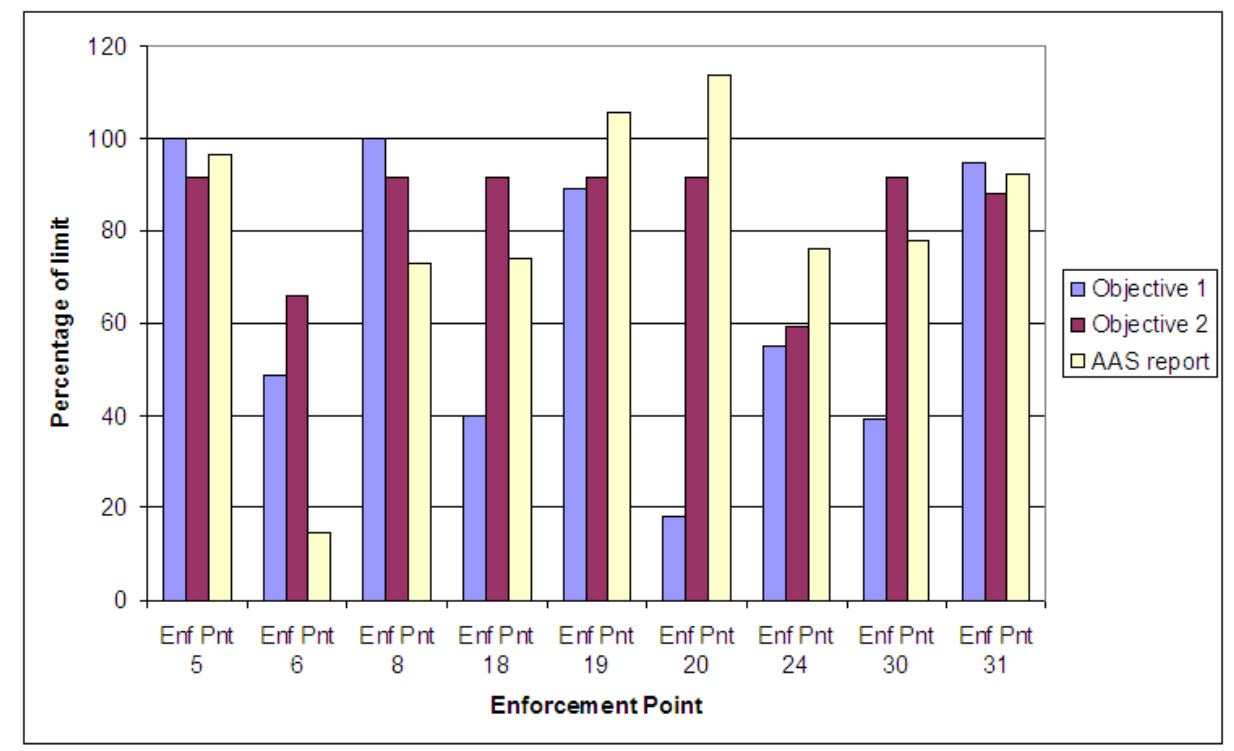

Figure 8: Comparison of results obtained for the two criteria, minimum average energy (Objective 1), and minimax (Objective 2) with the actual realized loads at Schiphol (AAS report)

Although the results obtained in the minimax optimization are in a much closer agreement with the actual results in comparison to the minimum average energy solution, the differences are still significant. As indicated earlier, this is likely in part due to the fact that in the minimax approach a variety of simplifying assumptions has been made, most notably the use of a limited number of enforcement points. Unlike for the actual result, the minimax solution provides a margin (in terms of energy) with respect to the noise limit of nearly $10 \%$ at 6 of the 9 enforcement points. The margin at the 3 remaining points is even larger.

\section{Conclusions}

The goal of this study was to develop a runway allocation optimization tool that seeks to maximize the permissible number of yearly flight movements within a specified noise budget. In this context a noise budget is to be understood as a set of regulations specifying that outside a certain surface area of land around the airport, community noise levels should not exceed certain specified annual limits. Although the runway allocation tool has 
been developed with a particular example airport in mind, viz., Schiphol airport, the model underlying the tool is sufficiently generic to permit application to other airports that are subject to similar noise budget restrictions. To enable an efficient use of the available noise budget, a minimax optimization model has been formulated that aims to reduce the risk of exceeding the noise limit at any point along the boundary of the protected area. At Schiphol airport, the noise budget is imposed at a discrete number of specified points, called enforcement points, located on the boundary contour of the protected area.

Due to the large-scale nature of the runway allocation planning problem, combined with limited computational resources, not all of the enforcement points were included in the optimization formulation presented in this study. Nevertheless, the number and distribution of the enforcement that were selected proved to be adequate to produce meaningful results. Indeed, the minimax optimization analysis resulted in a rather efficient noise distribution, reducing the maximum noise energy at any of the enforcement points included in the optimization formulation to a level some $10 \%$ below the permissible value, without overloading the enforcement points that were not included at the optimization analysis. Future research will focus on the development of a modeling approach that allows for more enforcement points to be taken into account at a reasonable computational cost, and that will include capacity constraints due to poor visibility conditions.

It needs to be noted that as of November 1, 2010, a new set of experimental noise regulations has been put into place at Schiphol airport, for the duration of a period of two years. The primary goal of the new set of regulations is to minimize the expected number of annoyed people, the same objective as used in the original SNAP tool that formed the basis for the present development. However, the existing regulations pertaining to the noise budget imposed at the enforcement points remains in principle intact. For this reason, the continued development of the minimax runway allocation optimization remains of considerable interest.

\section{References}

${ }^{1}$ Visser, H.G., Hebly, S.J., and Wijnen, R.A.A., Management of the Environmental Impact at Airport Operations, Nova Science Publishers, 2009

${ }^{2}$ Graham, A, Managing Airports: An International Perspective, Butterworth-Heinemann, 2008.

${ }^{3} \mathrm{http}: / /$ www.boeing.com/commercial/noise/listcountry.html (accessed 17 November 2010).

${ }^{4} \mathrm{http}: / /$ www.airports.org/aci/aci/file/ACI Priorities/Environment/TRACKER\%20FILE Airport\%20environment \%20initiatives.pdf (accessed 17 November 2010).

${ }^{5}$ Galis, S.P., Brouwer, M.A., and Joustra, T., "Optimization of yearly airport capacity within noise limits at Schiphol airport", 33rd International Congress and Exposition on Noise Control Engineering Internoise 2004, Prague, Czech Republic, August 2004.

${ }^{6}$ Kuiper B.R., Environmental Optimization of Runway Allocations - A Stochastic Repetitive Planning Approach, MSc thesis, Delft University of Technology, Delft, the Netherlands, March 2010.

${ }^{7}$ Heblij, S. J., and Wijnen, R. A. A.. "Development of a runway allocation optimisation model for airport strategic planning," Transportation Planning and Technology, 31(2), 2008, pp. 201-214.

${ }^{8}$ De Neufville, R., and Odoni, A.R., "Airport Systems: Planning, Design and Management”, McGraw-Hill, 2003.

${ }^{9}$ Sahai, Improved modeling of runway combination selection for noise management at Schiphol airport, MSc thesis, Delft University of Technology, Delft, the Netherlands, July 2009.

${ }^{10}$ Hillier, F., \& Lieberman, G. Introduction to Operations Research, McGraw-Hill, 2005.

${ }^{11}$ Olmstead, J., Fleming, G., and Gulding, J. Integrated Noise Model Version 6.0 User's Guide, ATAC Corporation, 1999.

${ }^{12}$ Ruijgrok, G., Elements of Airplane Performance, Delft University Press, 1996.

${ }^{13}$ ILOG, CPLEX release 9.1, 2005. 